\title{
SONOLUMINESCENT TOMOGRAPHY
}

\author{
Lihong V. Wang* and Qimin Shen \\ Optical Imaging Laboratory, Biomedical Engineering Program \\ Texas A\&M University, College Station, Texas 77843-3120, USA \\ Email: LWang@tamu.edu \\ URL: http://biomed.tamu.edu/ /w \\ * Corresponding author.
}

\begin{abstract}
A novel optical imaging technique called sonoluminescent tomography (SLT) was developed for crosssectional imaging of strongly scattering media noninvasively. Sonoluminescence, which was generated internally in the medium by continuous-wave ultrasound, was used to produce a two-dimensional image of an object embedded in a scattering medium by raster scanning the medium. The image had a high contrast and good spatial resolution. The spatial resolution was limited by the focal spot size of the ultrasound and can be improved by tightening the focus. This inexpensive imaging technique has potential applications in medicine and other fields related to scattering media.
\end{abstract}

Keywords - sonoluminescence, ultrasound, optical imaging, optical tomography, turbid media.

\section{INTRODUCTION}

Optical tomography of scattering media such as biological tissues is an increasingly active field because of its advantages in noninvasion, nonionization, and optical contrast for biomedical diagnosis.' Several optical imaging techniques being investigated include time-resolved optical imaging, frequency-domain optical imaging, optical coherence tomography, photo-acoustic tomography, and ultrasoundmodulated optical tomography. The imaging contrast is based on the difference in optical properties between abnormal and the surrounding normal biological tissues. All of these approaches use an external light source and overcome the light scattering problem to obtain optical images of scattering media.

We report, what is to our knowledge for the first time, a novel optical imaging technique called sonoluminescent tomography (SLT). ${ }^{2}$ SLT is based on light internally generated by ultrasound. The ultrasonic generation of light known as sonoluminescence (SL) was first reported in $1934,{ }^{3}$ which was multiple-bubble sonoluminescence (MBSL). SL has attracted an extraordinary amount of attention in this decade since single-bubble sonoluminescence (SBSL) was reported in $1990 .^{4-12}$ Although the full explanation of SL is still in development, it is well known that light is emitted when tiny bubbles driven by ultrasound collapse. The bubbles start out with a radius of several microns and expand to $\sim 50$ microns due to a decrease in acoustic pressure in the negative half of a sinusoidal period. After the sound wave reaches the positive half of the period, the situation rapidly changes. The resulting pressure difference leads to a rapid collapse of the bubbles accompanied by emission of light. The flash time of SL was measured to be in the tens of picoseconds. ${ }^{4}$ SBSL is so bright that it can be seen by the naked eye even in a lighted room, whereas MBSL is also visible in a darkened room. ${ }^{9}$ Researchers have envisioned possible applications of SL in sonofusion, sonochemistry, and building ultrafast lasers using the ultrafast flash of light in SL.

SLT is a new application of SL. In SLT, minimally scattering ultrasound is used to image optically scattering media. SLT contains information not available in the traditional ultrasonography. The major advantages of SLT include: 1) high signal-to-noise ratio due to the internally generated SL signal; 2) high imaging contrast; 3) good spatial resolution, which is scaleable with the ultrasonic focal size; and 4) low cost: the cost of equipment to generate SL is as low as hundreds of U.S. dollars. ${ }^{13}$

\section{METHODS AND MATERIALS}

A scattering medium was prepared by mixing $8.5 \mathrm{ml}$ of Intralipid (20\%) and $3.5 \times 10^{-7} \mathrm{Mol}$ of dominantly absorbing Trypan Blue dye (Sigma, T5526) in $370 \mathrm{ml}$ of distilled water. The reduced scattering coefficient and the absorption coefficient were $8.5 \mathrm{~cm}^{-1}$ and $0.017 \mathrm{~cm}^{-1}$ at $400 \mathrm{~nm}$, respectively. The scattering property is comparable with those of biological tissues. ${ }^{14}$

The scattering solution was contained in a $400-\mathrm{ml}$ beaker and was held on an $x-y$ translation stage (Fig. 1). A pink rubber cube was buried in the center of the solution. The ultrasonic transducer was adjusted such that the focal spot of the ultrasonic wave was at the center of the buried cube. The distance between the bottom of the ultrasonic transducer and the center of the cube was $3.5 \mathrm{~cm}$. The distance between the center of the cube and the bottom of the beaker was $4.0 \mathrm{~cm}$. The motorized translation stage, controlled by a personal computer (PC), was able to scan along both the $x$ and $y$ axes, which formed a plane perpendicular to the ultrasonic axis. An ultrasonic transducer (Panametrics, V314-SU) with a focal length of $3.68 \mathrm{~cm}$, a focal diameter of $0.3 \mathrm{~cm}$, and a focal zone of $3.44 \mathrm{~cm}$ transmitted vertically an ultrasonic wave into the scattering medium. The ultrasonic transducer was driven by an amplified $1-\mathrm{MHz}$ sinusoidal signal from a function generator (Stanford Research System, DS345). The amplification was achieved using a power amplifier (MiniCircuits, TIA-1000-1R8) and a transformer. The SL signal was detected by a photomultiplier tube (PMT) (Hamamatsu, R928) beneath the beaker, was differentially amplified by a 
low noise preamplifier (Stanford Research System, SR560), and was recorded using a digital oscilloscope (Tektronix, TDS 640A). Finally, the PC acquired the data from the oscilloscope through a GPIB interface.

\section{RESULTS AND DISCUSSION}

To measure the SL signal emanating from the scattering medium, a sensitive PMT was used. After background subtraction by differential amplification, the SL intensity was recorded as a DC voltage. The DC voltage represented the time-averaged SL intensity with the time constant of the detection system being $\sim 10 \mathrm{~ms}$. The maximum DC signal was $4.6 \mathrm{~V}$. The gain of the preamplifier was set to $5 \times 10^{4}$. The DC voltage across the PMT was $-986 \mathrm{~V}$. Based on the photocathode radiant sensitivity of $70 \mathrm{~mA} / \mathrm{W}$ at the $400 \mathrm{~nm}$ wavelength, the total luminescence power received by the $1.92 \mathrm{~cm}^{2}$ photocathode was estimated to be and $1.3 \times 10^{-15} \mathrm{~W}$.

While raster scanning the beaker in the $x-y$ plane with a step size of $1 \mathrm{~mm}$, the PC recorded the DC signals of SL versus the values of $x$ and $y$. The optical and ultrasonic systems were fixed while the beaker was scanned. A twodimensional image of the cubes buried in the scattering solution was plotted using the acquired data (Fig. 2). It is clearly seen from the images that the contrast was excellent, and the spatial resolution was $\sim 2-3 \mathrm{~mm}$. The contrast of the SL images was based on the difference between the optical and ultrasonic properties of the objects and those of the surrounding medium. These objects were optically opaque and ultrasonically absorbing. When the object was moved toward the ultrasonic focus, the SL intensity dropped quickly for several reasons. Firstly, the ultrasonic field was reduced at and below the focus. Secondly, the object yielded no SL signal. Thirdly, the SL signal above the object was partially blocked by the object.

Although the object used in this experiment had both optical and ultrasonic contrast relative to the background medium, images of objects may be obtained using SLT based

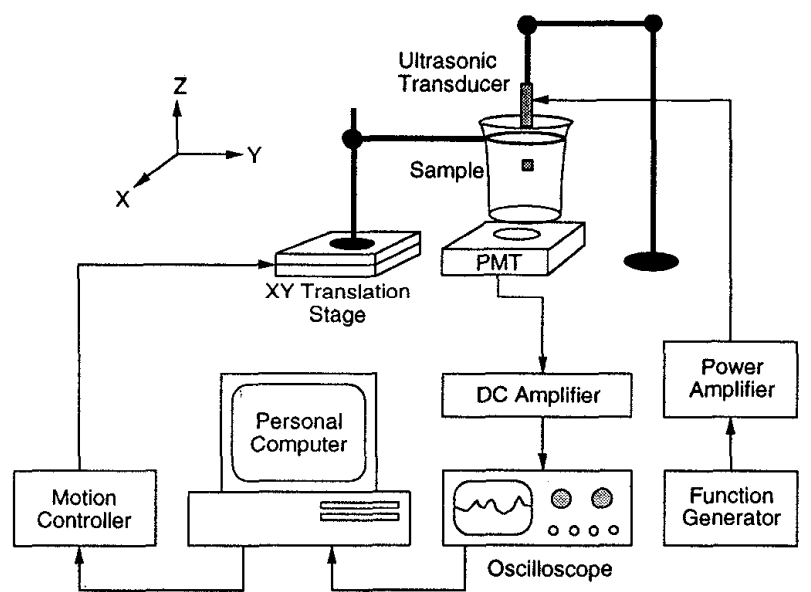

Fig. 1. Schematic diagram of the experimental setup for imaging scattering media using sonoluminescence. on several contrast mechanisms in general. When an object has contrast in SL generation, the SL signal originating from the object will differ from that originating from the background medium. The SL generation may be affected by the local ultrasonic intensity or the local concentration of SL enhancing chemicals if they are used. When an object has contrast in optical absorption coefficient, scattering coefficient, or scattering anisotropy, the SL signal from the object will be attenuated differently because the SL light must propagate through the object.

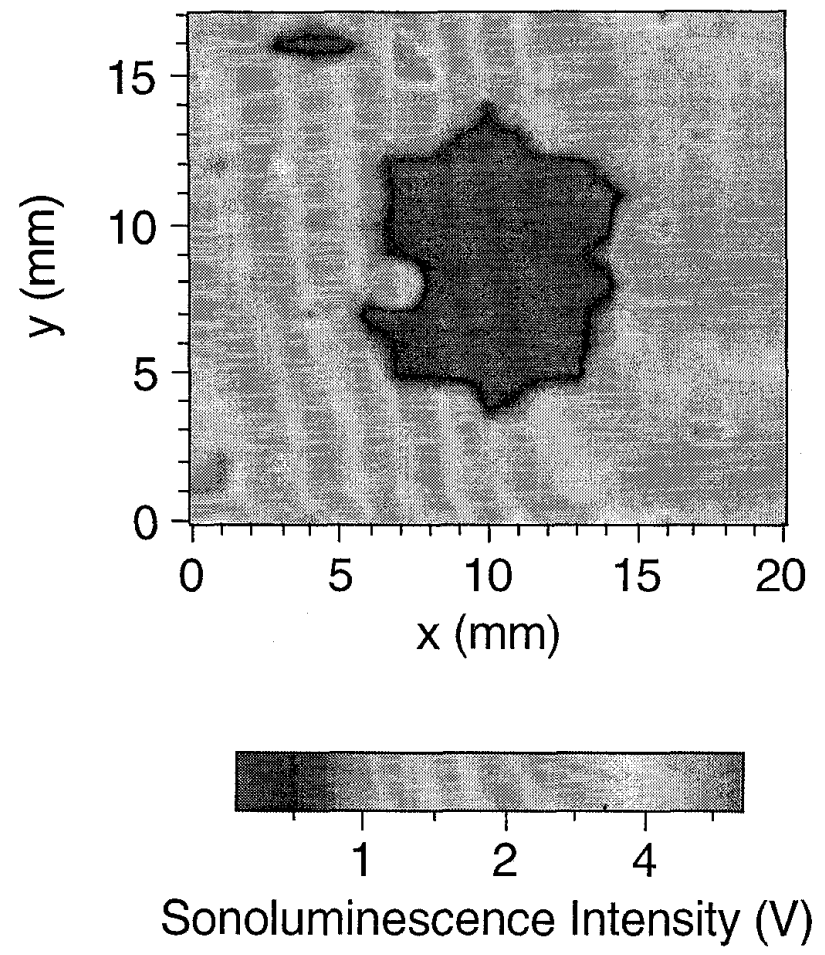

Fig. 2. Two-dimensional SLT image of an object buried in a turbid solution.

Using the present ultrasonic system, we obtained a SL column of $-3.5 \mathrm{~cm}$ in length and $\sim 0.3 \mathrm{~cm}$ in diameter. The length of the SL column limits the imaging resolution along the ultrasonic axis. Similarly, the diameter of the SL column determines the imaging resolution on the $x-y$ plane. A more tightly focused ultrasonic transducer may be used to reduce the size of the SL column significantly. When the SL column is reduced to a desired size, one may acquire 3-dimensional images of scattering media by scanning in all three directions.

SL light propagates outward in the scattering medium in all directions. We may increase the signal-to-noise ratio of the detection system dramatically by integrating the SL light over a large detection area.

Although there are potentially harmful effects caused by cavitation, the threshold of ultrasound intensity leading to volume lesions is very high. The damage threshold in spatialpeak-temporal-peak (SPTP) power was reported to be 400 $\mathrm{W} / \mathrm{cm}^{2}$ and $900 \mathrm{~W} / \mathrm{cm}^{2}$ at $1 \mathrm{MHz}$ for dog brain tissue and $\mathrm{dog}$ 
thigh muscle, respectively. ${ }^{15}$ The peak pressure in our experiment was -2.5 bars at the ultrasonic focus, corresponding to an SPTP power of $2.1 \mathrm{~W} / \mathrm{cm}^{2}$, which was two orders of magnitude less than the damage threshold. The peak pressure was also far less than the 23-bars safety limit set by the U.S. Federal Drug Administration, which is usually conservative. ${ }^{16}$

\section{CONCLUSIONS}

In conclusion, we developed and demonstrated a novel optical tomography technique using SL. Both the spatial resolution and the contrast of the images were very good and may be improved further. The SLT technique is expected to find applications in biomedicine and other fields involving highly scattering media.

\section{ACKNOWLEDGMENT}

Thanks to $\mathrm{X}$. Zhao for her experimental assistance in electronics. This project was sponsored by the National Institutes of Health grants R29 CA68562 and R01 CA71980 and a Whitaker grant.

\section{REFERENCES}

1. R. R. Alfano and J. G. Fujimoto, editors, Advances in Optical Imaging and Photon Migration, TOPS Vol. 2 (Optical Society of America, Washington, D. C., 1996).

2. L.-H. V. Wang and Q. Shen, Optics Letters 23, 561-563 (1998).

3. H. Frenzel and H. Schultes, Z. Phys. Chem. B 27, 421 (1934).

4. B. P. Barber and S. J. Putterman, Nature 352, 318 (1991).

5. E. B. Flint and K. S. Suslick, J. Phys. Chem. 95, 1484 (1991).

6. L. A. Crum and S. Putterman, J. Acoust. Soc. Am. 91, 517 (1992).

7. C. C. Wu and P. H. Roberts, Phys. Rev. Lett. 70, 3424 (1993).

8. W. C. Moss, D. B. Clarke, J. W. White, and D. A. Young, Phys. Fluids 6, 2979 (1994).

9. L. A. Crum and R. A. Roy, Science 266, 233 (1994).

10. C. Eberlein, Phys. Rev. Lett. 76, 3482 (1996).

11. J. B. Young, T. Schmiedel and W. Kang, Phys. Rev. Lett. 77, 4816 (1996).

12. B. P. Barber, R. A. Hiller, R. Löfstedt, S. J. Putterman, and K. R. Weninger, Phys. Reports 281, 65 (1997).

13. R. A. Hiller and B. P. Barber, Scientific American 272, 96 (February 1995).

14. W. F. Cheong, S. A. Prahl, and A. J. Welch, IEEE J. Quantum Electronics 26, 2166 (1990).

15. F. J. Fry, N. T. Sanghvi, R. S. Foster, R. Bihrle, and C. Hennige, Ultrasound Med. Biol. 21, 1227 (1995).

16. T. A. Whittingham, Imaging 6, 33 (1994). 